\title{
A Hierarchical Subpopulation Particle Swarm Optimization Algorithm
}

\author{
Chuan Lin Quanyuan Feng \\ School of Information Science and Technology, Southwest Jiaotong University, Chengdu 610031, P. R. China
}

\begin{abstract}
Based on the metaphor of specialization and cooperation in hierarchical social organization, a new particle swarm optimization (PSO) algorithm, hierarchical subpopulation PSO (HS-PSO), is proposed. In HS-PSO, the entire population is divided into several subpopulations which are arranged in a hierarchy. The subpopulations at the same level of the hierarchy evolve relative independently and cooperate with each other via their respective best particles. For the particles at different levels, different tasks are assigned. Two versions of HS-PSO which use the same or different kinds of PSO algorithms for the particles at different levels are presented. The efficiency of HS-PSO is verified by the simulation results.
\end{abstract}

Keywords: Particle swarm optimization, Hierarchy, Subpopulation, Specialization and Cooperation

\section{Introduction}

The particle swarm optimization (PSO) is a new global optimization method based on a metaphor of social interaction [1]-[2]. Since presented, it has found applications in wide areas [3]. The traditional PSO uses a kind of neighborhood topology known as gbest, in which all particles are connected and directly influenced by the best particle in the entire population. It has been thought that gbest type PSO converges quickly at the early stage but has a weakness of premature convergence. To increase the diversity of the particle swarm and avoid premature convergence, many researchers have investigated other kinds of topologies [4]-[5]. In [6], a hierarchical particle swarm (H-PSO) algorithm has been presented, in which the particles are arranged in a dynamic hierarchy that is used to define a neighborhood structure. In [7], a multi-swarm cooperative particle swarm optimizer (MCPSO) based on a master-slave model was presented. In MCPSO, the diversity of the particle swarm is amplified by the independent evolution of the slave swarms. In this paper, we propose a new PSO algorithm, hierarchical subpopulation PSO (HS-
PSO), based on the metaphor of specialization and cooperation in hierarchical human society or organization. In HS-PSO, the population is divided into several subpopulations which are arranged as a hierarchy. The non-best particles in a subpopulation evolve independently while the best particle composes the subpopulation at upper level and evolves there. Different inertial weights are used at different levels of the hierarchy for better balance of exploration and exploitation. Two versions of HS-PSO which use the same or different kinds of PSO algorithms for different levels are presented. The simulation results of several benchmark functions show that HS-PSO evidently improves the PSO algorithm.

\section{PSO algorithm and related works}

PSO is a population-based iterative optimization method which finds the solution to a problem in $D$ dimensional space by a swarm of $m$ particles. At each iteration, the velocity and position of the ith particle are updated by the following formulas[2]:

$$
\begin{gathered}
v_{i d}(t+1)=w v_{i d}(t)+c_{1} r_{1 d}(t)\left(p_{i d}-x_{i d}(t)\right)+c_{2} r_{2 d}(t)\left(p_{g d}-x_{i d}(t)\right) \\
x_{i d}(t+1)=x_{i d}(t)+v_{i d}(t+1)
\end{gathered}
$$

Where, $i=1, \ldots, m, d=1, \ldots, D . x_{i}(t)$ and $v_{i}(t)$ are the position and velocity of particle $i$ at time step $t$, respectively, $\boldsymbol{p}_{i}$ is the personal best position of particle $i, \boldsymbol{p}_{g}$ is the best position found in the neighborhood of particle $i . w$ is inertia weight, $c_{1}, c_{2}$ are acceleration coefficients. $r_{1 d}(t), \quad r_{2 d}(t)$ are random numbers uniformly distributed in $[0,1]$. In the first part of Eq. (1), inertial weight $w$ determines how much of the particle's previous velocity is preserved. The second part is referred to as "cognitive" part, which represents the private thinking of the particle itself. And the third part is the "social” part, which represents particle's learning from the elite particle in the society.

From Eq. (1), we see that $\boldsymbol{p}_{g}$ and $\boldsymbol{p}_{i}$ actually play a role of attractors in PSO algorithm. For standard PSO with gbest topology, all the particles are strongly attracted by $\boldsymbol{p}_{g}$, which may cause premature convergence if $\boldsymbol{p}_{g}$ is stagnant at the early stage. Various methods have been designed to increase the 
diversity of the particle swarm. In [4], many kinds of neighborhood topologies for the PSO algorithm have been studied. And the simulation results show that the von Neumann neighborhood topology performs well, while the commonly used gbest, lbest and star topologies have been rated worse. In the hierarchical PSO (H-PSO) algorithm [6], the particles are arranged in a hierarchy and the neighborhoods are constantly changing. This is helpful to prevent premature convergence. In [8], the fully informed particle swarm (FIPS) algorithm has been presented. In the FIPS, particle $i$ employs all its neighbors' personal best positions to adjust its velocity[9]:

$$
v_{i d}(t+1)=w v_{i d}(t)+\sum_{n=1}^{N_{i}} \frac{c r(t)\left(p_{\mathrm{nbr}(n) d}-x_{i d}(t)\right)}{N_{i}}
$$

Where $N_{i}$ is the number of particle $i$ 's neighbors and $\operatorname{nbr}(n)$ is the index of particle $i$ 's $n$th neighbor. By including more neighbors to update the velocity, the diversity of FIPS is increased. In [7], a multi-swarm cooperative particle swarm optimizer (MCPSO) inspired by the phenomenon of symbiosis in natural ecosystems has been presented. MCPSO is based on a master-slave model, which uses several independently evolving salve swarms to maintain the diversity and one master swarm to speed up the convergence by sharing the information of the most successful particles in the slave swarms.

\section{Hierarchical subpopulation PSO}

As PSO algorithm is originally developed from the simulation of social behavior, it may be further improved by emulating some efficient social organizing and learning mechanism. In the human society or business organization, people are often divided into several groups or departments for special tasks and organized as a hierarchy with the elites at upper level. People in different groups work relatively independently and cooperate with each other via the communication of the leaders (elites) in the groups. Based on this metaphor, we propose a new PSO algorithm, hierarchical subpopulation PSO (HS-PSO). In HS-PSO, the entire population is divided into several subpopulations, which form the bottom layer of the given hierarchy. The non-best particles in each subpopulation evolve independently to keep a good diversity of the whole population, while the best particles of the subpopulations compose the new subpopulations at upper level of the hierarchy for fast evolution. Thus, both good diversity and fast speed can be obtained in HS-PSO by proper balance of exploration and exploitation. For the subpopulations at upper level, the non-best and best particles evolve similarly. Note that for the subpopulation at the top level, all the particles including the best particle evolve in it.

Now we take HS-PSO with two-level hierarchy (see fig. 1) as an example to describe HS-PSO algorithm in detail. First, the entire population of size 20 is divided into 4 subpopulations of size 5: Sub21, Sub22, Sub23 and Sub24. At each iteration, the nonbest particles in subpopulations Sub21 Sub24 are first updated with a single PSO or its variant independently, while the best particles in the 4 subpopulations are submitted to compose the upper subpopulation Sub1 of size 4. Note that the best particles in Sub21 Sub24 belong to both Sub21 Sub24 and Sub1. For the toplevel subpopulation Sub1, its all particles are updated there.

For HS-PSO with hierarchy having more than two levels, the subpopulation can be updated from low to above of the hierarchy similarly as above. In fig. 2, a population of size 20 is divided into 4 subpopulations of size 5: Sub31, Sub32, Sub33 and Sub34. Subpopulation Sub21 of size 2 at the middle level is composed of the best particles of Sub31 and Sub32. Sub1 of size 2 is composed of the best particles of Sub21 and Sub22. In each subpopulation except the top-level subpopulation Sub1, only its non-best particles are updated. In Sub1 all the particles are updated there.

In sum, the general steps for HS-PSO can be described as follows:

Step 1: Initialize all the particles' positions and velocities in the entire population and divide the population according to the given hierarchy.

Step 2: Evaluate all the particles' fitness values.

Step 3: Update all the particles' personal best positions and global best position.

Step 4: FOR each level of the hierarchy (from low to above)

FOR each subpopulation at a level

IF the subpopulation is not at the toplevel

Step 4a: Summit the best particle to compose the upper subpopulation according to the hierarchical structure.

Step 4b: Update the non-best particles' positions and velocities in the subpopulation with a kind of PSO algorithm (e.g. Eq. (1) and (2)).

IF the subpopulation is at the top-level

Step 4c: Update all the particles' positions and velocities 
in the subpopulation with a kind of PSO algorithm.

Step 5: If the ending condition is met, end the algorithm, otherwise return to step 2.

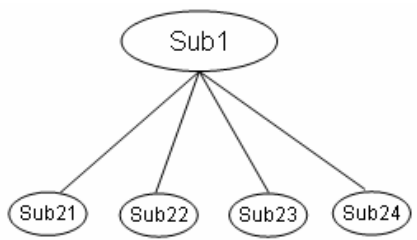

Fig. 1: 2-level hierarchy of subpopulations.

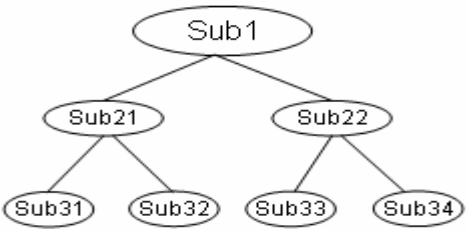

Fig. 2: 3-level hierarchy of subpopulations.

In general, highly interconnected populations have quick communication but may give rise to premature convergence. On the other hand, populations with few connections help to prevent premature convergence by inhibiting communication but may cause the particles to wander around some unpromising regions for a long time. In HS-PSO, the information is flowed from the upper level to the lower level of the hierarchy gradually, and the communication in the particle swarm is moderate and suitable for a proper balance of global and local searching ability. As the subpopulation at upper level is composed of the varying best particles in different subpopulations at lower level, the particles at the same upper level generally have comparable performance and good diversity with dynamic neighbors. Hence the cooperation between these particles is more effective. To further improve HS-PSO, various tasks are associated to the particles at different levels of the hierarchy since the particles at different levels have distinct performance. The particles at upper level mainly focus on exploration while the particles at lower level mainly focus on exploitation. Accordingly, the larger inertial weight is used at higher level. The inertia weight $w_{k}$ at level $k$ can be given by Eq. (4):

$$
w_{k}=w_{\max }-\frac{(k-1)\left(w_{\max }-w_{\min }\right)}{h-1}
$$

We suppose that the top level is level 1, which uses maximum inertial weight $w_{\max }$, and the bottom level uses minimum inertial weight $w_{\min }$. The idea for this strategy is that the non-best particles in the lower subpopulations will move towards the best particles and search around them, thus the best particles should be active to explore new better solutions in order to give positive influence on the non-best particles. If the best particle of a subpopulation converges too early, the population tends to be premature easily. So we let the particles at upper level have larger inertial weight $w$ to explore new regions while the particles at lower level have smaller $w$ to exploit the prominent regions.

For different subpopulations different PSO algorithms can be used. In this paper, two versions of HS-PSO are proposed. The first version (denoted as HSPSO-S) uses standard PSO algorithm in all the subpopulations. The second version (denoted as HSPSO-F) uses standard PSO algorithm for the bottom level but FIPS algorithm for upper levels. HSPSO can still be extended by submitting several elite particles rather than the best particle in a subpopulation to compose the upper subpopulation.

\section{Simulation and results}

In this section, we employ five benchmark functions to test the performance of HSPSO-S and HSPSO-F and compare them with PSO with gbest topology (PSO-g) and von Neumann topology (PSOvon). These functions include Sphere, Rosenbrock, Rastrigin, Griewank and Ackley[6], all with dimension $D=30$. The first two functions, Sphere and Rosenbrock, are unimodal functions while the remaining three functions are complex multimodal functions. The search range $\left[X_{\min }, X_{\max }\right]^{D}$ for each benchmark function are showed in table 1 . In our experiments, 20 particles have been used. The initial range of the particles' positions is $\left[0, X_{\max }\right]^{D}$, and the initial velocities are in $\left[-V_{\max } / 2, V_{\max } / 2\right]^{D}$, where the maximum velocity $V_{\max }=0.5\left(X_{\max }-X_{\min }\right)$. In PSO-g and PSOvon, $w=0.729, c_{1}=c_{2}=1.494$. For HSPSO-S and HSPSO-F, the hierarchy shown in fig. 1 is used, and $c_{1}=c_{2}=1.78$, the inertial weight $w$ is decided by Eq. (4), where $w_{\max }=0.85, w_{\min }=0.5$. In HSPSO-F, $\mathrm{c}=1.78 * 2=3.56$ for FIPS at upper level. Each run has been repeated 50 times. The maximum iteration for each run is set as 3000 . The mean best fitness value and standard variation for 4 PSO algorithms after 3000 iterations are shown it tab. 2 . And the convergence of mean best fitness value, in the form of logarithm with base 10 , for 5 benchmark functions are shown in fig. 3 7.

\begin{tabular}{|c|c|}
\hline functions & {$\left[X_{\min }, X_{\max }\right]^{D}$} \\
\hline Sphere & {$[-100,100]^{D}$} \\
\hline Rosenbrock & {$[-30,30]^{D}$} \\
\hline Rastrigin & {$[-5.12,5.12]^{D}$} \\
\hline Griwank & {$[-600,600]^{D}$} \\
\hline Ackley & {$[-32,32]^{D}$} \\
\hline
\end{tabular}

Tab. 1: Search range for five benchmark functions. 


\begin{tabular}{|c|c|c|}
\hline \multirow{2}{*}{ functions } & \multicolumn{2}{|c|}{$\begin{array}{c}\text { Mean best fitness value } \\
\text { (standard variation) }\end{array}$} \\
\cline { 2 - 3 } & PSO-g & PSOvon \\
\hline Sphere & $4.60 \mathrm{e}-23(2.52 \mathrm{e}-22)$ & $3.12 \mathrm{e}-31(1.03 \mathrm{e}-30)$ \\
\hline Rosenbrock & $37.60(42.18)$ & $47.68(43.25)$ \\
\hline Rastrigin & $105.57(18.93)$ & $90.54(20.74)$ \\
\hline Griwank & $0.1527(0.3574)$ & $0.0138(0.0170)$ \\
\hline Ackley & $3.9970(2.1699)$ & $0.7522(0.9082)$ \\
\hline
\end{tabular}

Tab. 2: Mean best fitness value and standard variation in five benchmark functions.

\begin{tabular}{|c|c|c|}
\hline \multirow{2}{*}{ functions } & \multicolumn{2}{|c|}{$\begin{array}{c}\text { Mean best fitness value } \\
\text { (standard variation) }\end{array}$} \\
\cline { 2 - 3 } & HSPSO-S & HSPSO-F \\
\hline Sphere & $1.93 \mathrm{e}-34(8.99 \mathrm{e}-34)$ & $4.05 \mathrm{e}-38(5.27 \mathrm{e}-38)$ \\
\hline Rosenbrock & $37.4693(31.3526)$ & $36.9355(29.3884)$ \\
\hline Rastrigin & $81.7827(16.9103)$ & $64.4330(15.5890)$ \\
\hline Griwank & $0.0059(0.0087)$ & $0.0033(0.0049)$ \\
\hline Ackley & $1.30 \mathrm{e}-14(3.37 \mathrm{e}-15)$ & $9.34 \mathrm{e}-15(2.68 \mathrm{e}-15)$ \\
\hline
\end{tabular}

Tab. 2 (continued)

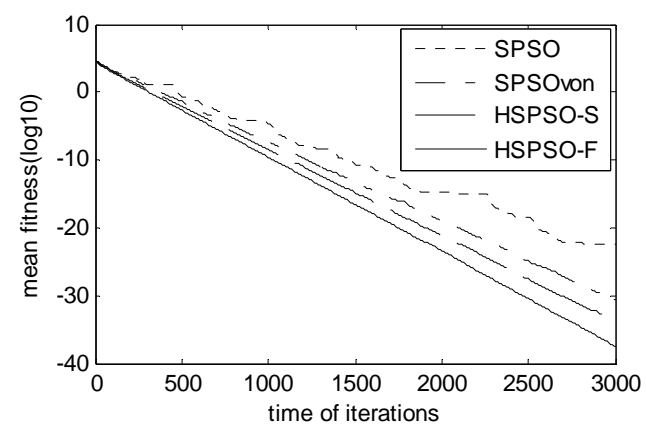

Fig.3: Convergence of mean best fitness for Sphere.

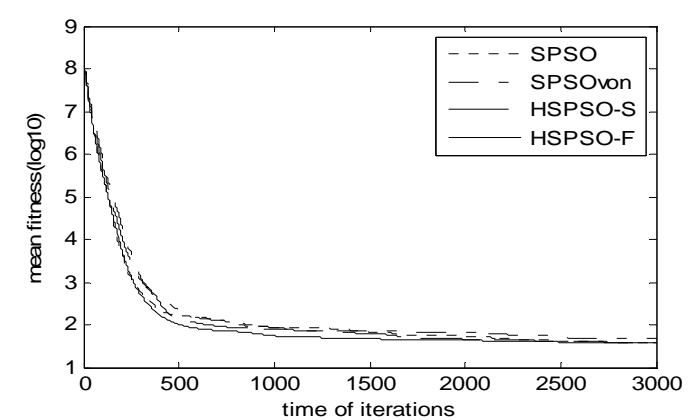

Fig.4: Convergence of mean best fitness for Rosenbrock.

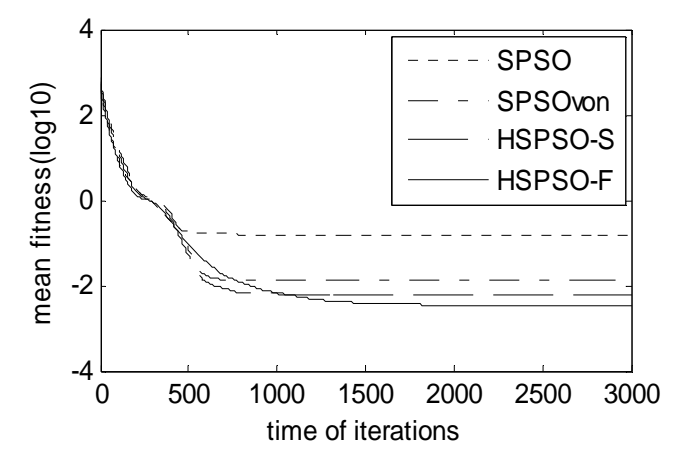

Fig.5: Convergence of mean best fitness for Rastrigin.

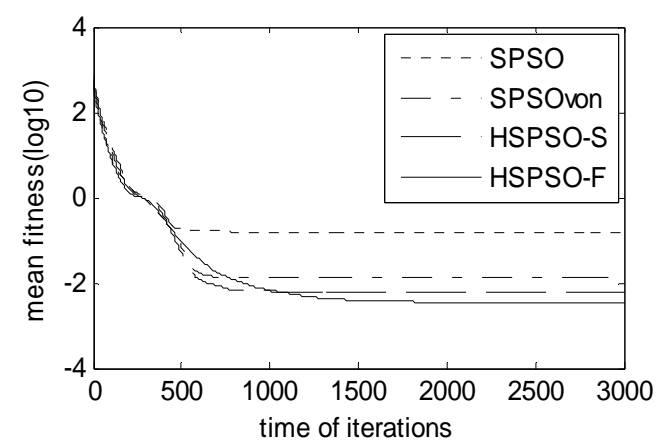

Fig.6: Convergence of mean best fitness for Griewank.

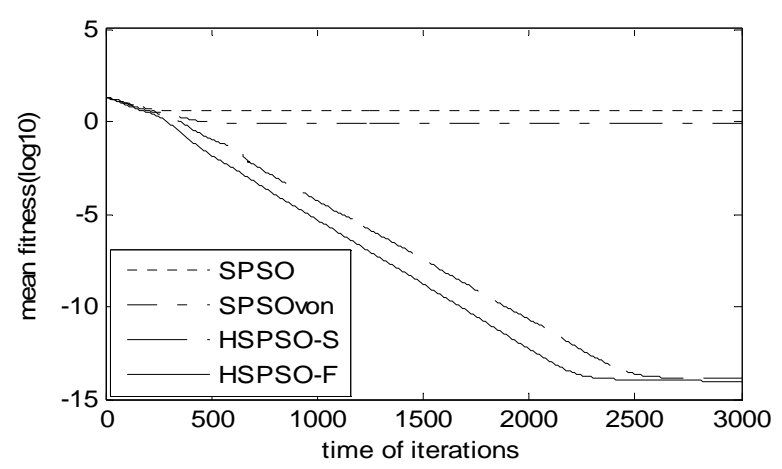

Fig.7: Convergence of mean best fitness for Ackley.

Table 2 shows that HSPSO-F achieves the minimum mean best fitness among the 4 PSO algorithms in all the 5 test functions and HSPSO-S ranks second. PSOvon gets smaller mean best fitness than PSO-g except in function Rosenbrock. From fig. 3 7, we can see that in functions Sphere, Rosenbrock and Ackley, HSPSO-F converges fastest and HSPSO$\mathrm{S}$ ranks second. In function Griewank, HSPSO-S converges fastest. And PSO-g converges fastest in function Rastrigin but stagnates in the early stage. It is obvious that HSPSO-S and HSPSO-F perform better than PSO-g and PSOvon due to the specialization and cooperation of the subpopulations in the hierarchy and a good balance of exploration and exploitation. As the particles at upper level have comparable performance and good diversity, each particle may provide some special information for others in the subpopulation. Hence employing FIPS algorithm at the upper level to make the particle share more information from its all neighbors seems a good strategy. This explains the reason that HSPSO-F performs best in the 4 PSO algorithms.

\section{Conclusions}

Based on the metaphor of specialization and cooperation and the hierarchical organization in human society, a novel PSO algorithm, hierarchical 
subpopulation PSO (HS-PSO) was presented in this paper. In HS-PSO, the entire population is divided into several subpopulations that are arranged in a hierarchy, and the exploration and exploitation ability can be well balanced by assigning different tasks to the particles at different levels. Two versions of HS-PSO algorithms are given in this paper: HSPSO-S which uses standard PSO algorithm in all the subpopulations and HSPSO-F which uses standard PSO algorithm for the subpopulations at the bottom level but FIPS algorithm for upper levels. The simulation results with a set of benchmark functions show that both HSPSO-S and HSPSO-F can evidently improve the performance of PSO algorithm and HSPSO-F performs best in both unimodal and multimodal functions. Our further study will focus on the following aspects to further extend HS-PSO:

(1) Employ adaptive hierarchical structure.

(2) Exchange the information between the subpopulations.

\section{References}

[1] J. Kennedy, R.C. Eberhart, Particle swarm optimization. Proceedings of the IEEE International Joint Conference on Neural Networks, pp. 1942-1948, 1995.

[2] Y. Shi, R. C. Eberhart, A modified particle swarm optimizer. Proceedings of the IEEE International Conference on Evolutionary Computation, pp. 69-73, 1998.

[3] A. Chatterjee, K. Pulasinghe, K. Watanabe, etc. , A Particle-Swarm-Optimized Fuzzy-Neural Network for Voice-Controlled Robot Systems. IEEE Trans. On Industrial Electronics, 52:14781489, 2005.

[4] J. Kennedy, R. Mendes, Population structure and particle swarm performance. CEC 2002, pp. 1671-1676, 2002.

[5] P. N. Suganthan, Particle swarm optimizer with neighborhood operator. CEC 1999, pp. 19581962, 1999

[6] S. Janson, M. Middendorf, A hierarchical particle swarm optimizer and its adaptive variant. IEEE Trans. On Systems, Man, and CyberneticsPart B: Cybernectics, 35: 1272-1282, 2005.

[7] B. Niu, Y. Zhu, X. X. He, etc., MCPSO: A multi-swarm cooperative particle swarm optimizer. Applied Mathematics and computation, 185: 1050-1062, 2007.

[8] R. Mendes, J. Kennedy, The fully informed particle swarm: simpler, maybe better. IEEE Trans. on Evolutionary Computation, 8: 204-210, 2004.
[9] J. Kennedy, R. Mendes, Neighborhood topologies in fully informed and best-ofneighborhood particle swarm. IEEE Trans. on Systems, Man, and Cybernetics-Part C: Applications and Reviews, 36: 515-519, 2006. 